\title{
Wearable Olfactory Display: Using Odor in Outdoor Environment
}

\author{
Tomoya Yamada* \\ Satoshi Yokoyama \\ Tomohiro Tanikawa \\ Koichi Hirota ${ }^{\S}$ \\ Michitaka Hirose \\ Graduate School of \\ Information Science and \\ IBM Japan, Ltd. \\ Research Center of \\ Graduate School of \\ Frontier Science, \\ Technology, \\ the University of Tokyo \\ Information Science and \\ Technology, \\ the University of Tokyo
Research Center of
Technology, \\ Information Science and \\ the University of Tokyo
}

\begin{abstract}
Recently, there are various types of display systems that can present aural, visual and haptic information related to the user's position. It is also important to present olfactory information related to the user's position, and we focus on the spatiality of odor, which is one of its characteristics. In this research, we constructed and evaluated a wearable olfactory display to present the spatiality of odor in an outdoor environment. The prototype wearable olfactory display system treats odor in the gaseous state, and the odor air is conveyed to the user's nose through tubes. Using this system, we also present the spatiality of odor by controlling the odor strength according to the positions of the user and the odor source. With this prototype system, the user can specify the position of the odor source in an outdoor environment. To improve this prototype system, we constructed another wearable olfactory display. Because odor is treated in the gaseous state, the first prototype system has some problems such as the large size of the device and unintentional leakage of the odor into the environment. To solve these issues, we developed and evaluated an advanced wearable olfactory display that uses an inkjet head device to treat odor in the liquid state.
\end{abstract}

CR Categories: H.5.1 [Information Interface and Presentation]: Multimedia Information Systems -Artificial, augmented, and virtual realities; C.5.3 [Computer System Implementation]: Microcomputers -Portable device

Keywords: sense of smell, olfactory information, wearable device

\section{INTRODUCTION}

The important point in using odor in virtual reality (VR) is how to control the sensory stimulation of odor temporally and spatially. In the real world, people can sense the spatial information of odor and identify the type of odor from the type of odor molecule and identify the placement of the odor source by perceiving the change of odor strength. These constitute the spatial information of odor. In the VR world, as in the real world, we can present the spatial information of odor by real-time control of odor stimulation depending on the user's movement. We are proposing the idea of an "odor field" as the mode of expression of the spatial information of odor in the VR space. We describe the spatial spread and variation with time of the type and strength of odor as

\footnotetext{
* tomoya@cyber.rcast.u-tokyo.ac.jp

$\dagger$ yoko@cyber.rcast.u-tokyo.ac.jp

+tani@cyber.rcast.u-tokyo.ac.jp

$\S$ hirota@cyber.rcast.u-tokyo.ac.jp

『 hirose@cyber.rcast.u-tokyo.ac.jp
}

IEEE Virtual Reality 2006

March 25 - 29, Alexandria, Virginia, USA

1-4244-0224-7/06/\$20.00 @2006 IEEE

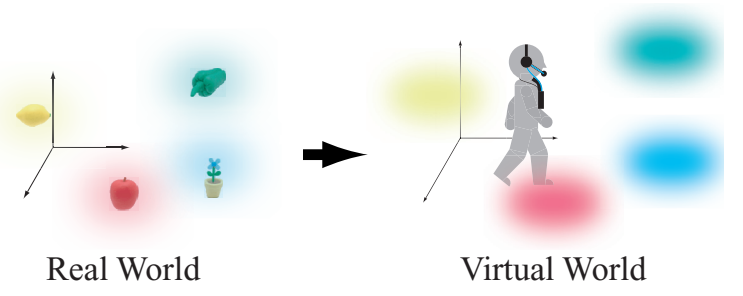

Fig.1 Concept of Odor Field

the density of odor molecules, and we called this the "odor field". The concept of the odor field as a situation in which several odor sources are arranged in space is shown in Fig. 1.

For presenting the odor field, we are developing an olfactory display which can present odor stimulation depending on the user's position which is detected by the spatial localization sensor. With previous olfactory displays, because the user's field of activities was limited, it was difficult to make full use of the characteristics of the olfactory sense such as odor source detection. Therefore, in this research, we are developing a wearable olfactory display which can present the odor field in various environments such as a wide space or outdoors. We constructed two prototype wearable olfactory displays: prototype one has tubes to convey the generated odor air to the user's nose, and prototype two has an inkjet head device to present a very small odor droplet directly to the user's nose. Also, to evaluate the applicability of presenting an odor field in a wide space, we developed a system that can present the odor field in a wide space by means of a wearable olfactory display.

\section{RELATED WORKS}

In engineering, there have been several approaches in the study of odor, such as the study of odor sensors, the study of the transport of odor information and the study of olfactory displays. There have also been similar attempts to present odor in entertainment and art.

\subsection{Odor Sensor}

Some odor sensors that react to characteristic chemical odor materials have been developed. These were mainly made of a semiconductor or crystal vibration chip. There is also a study on the reproduction of an identifiable odor type using multiple odor sensors with different characteristics [1,2,3]. Currently because no standard odor types analogous to RGB in optics have been found, it is impossible to develop a sensing system that can identify all odor types [4].

\subsection{Olfactory Display}

The development of an odor display system that can present odor selectively began in the early days. The oldest system is "Sensorama" [5]. "Sensorama" is a game machine, wherein the 
chair or steering wheel vibrates and odor is presented according to the scene on the display. In the AMLUX Theater, there was an attempt to add odor information to visual media such as movies. There were discussions about which movie scene needs odor presentation, referring to experiments on presenting odor synchronously with the movie. In arts, there have been tests to induce the effect of relaxation through odor presentation. Also there were some approaches to use odor information in training programs, such as the fire-fighter training system called "FiVe FiRe"[6].

Some odor presentation systems for supplementing odor information to Web and KIOSK terminals have been developed $[7,8,9,10]$. These systems present odor information by evaporating perfumed material or by spraying it using propellant gas or inkjet technology. Odor added to advertisements shown on visual displays presents important information, not only on how a product looks but also on how it smells.

Additionally, the development of odor blending devices which can accurately identify the type of odor and reproduce that odor type is in progress. The odor blending device blends several perfumes based on the response pattern of the odor sensing array to reproduce the intended odor [11]. Through the development of this kind of system, the study of odor recording and odor reproduction systems is progressing [12]. There also are studies on transporting the spatial information of odor. There is a system being developed for presenting spatial odor information to a distant location by transporting data on the type and strength of odor detected by odor sensors [13]. Furthermore, there is the idea that tele-existance with highly realistic sensation can be made possible by assembling odor sensors as well as cameras on a humanoid robot.

\subsection{Presentation of Spatial Information of Odor}

There are two types of olfactory displays which can present the spatial information of odor: one is the olfactory display worn by the user (we called it the wearable type) and the other is the olfactory display installed in the environment (we called it the ubiquitous type). One idea for a wearable-type olfactory display is a system with a spatial position sensor (Polhemus Inc., FASTRAK) that detects the position of the user's nose, the data of which is used to calculate the strength of the odor air presented through a silicon tube to the user's nose [14]. However, this system has a large odor-generating part to control the odor strength, and a limited odor presentation area because of the length of the silicon tube. For the ubiquitous-type olfactory display, a system that fires a mass of odor air to the user's nose that is detected by a camera system has been proposed [15]. This system is also difficult to use in a large space, such as outdoors, because of its limited firing range and susceptibility to environmental factors, such as wind, and clogging.

In this research, we adopted the wearable-type olfactory display, because our olfactory display must be robust against the influence of the environment and accurate in the presentation of odor strength. This type of olfactory display can present odor according to the user's position by using a spatial positioning sensor. In this work, we developed an olfactory display that can be used in large spaces, including outdoor environments in which the user can walk around freely, by miniaturization and weight reduction of the olfactory display and by making it wearable.

\section{Wearable Olfactory Display With Tubes for Presentation (Prototype 1)}

We constructed a prototype wearable olfactory display. This wearable olfactory display is made up of the odor-generating unit,

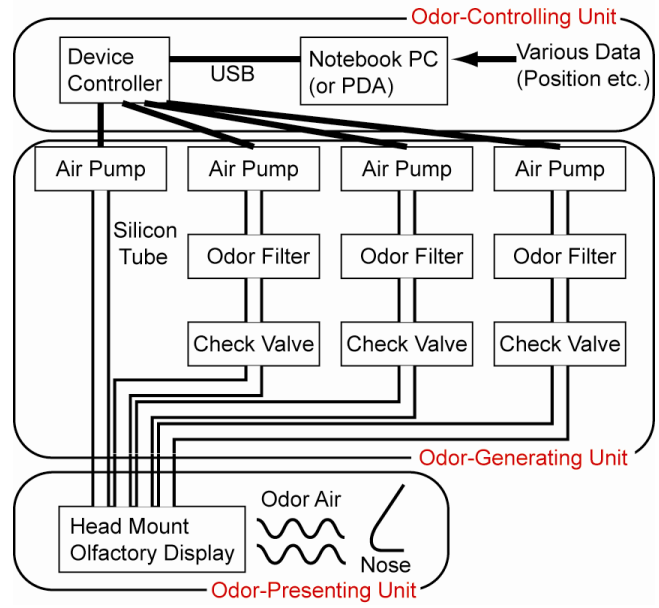

Fig.2 Configuration of Wearable Olfactory Display

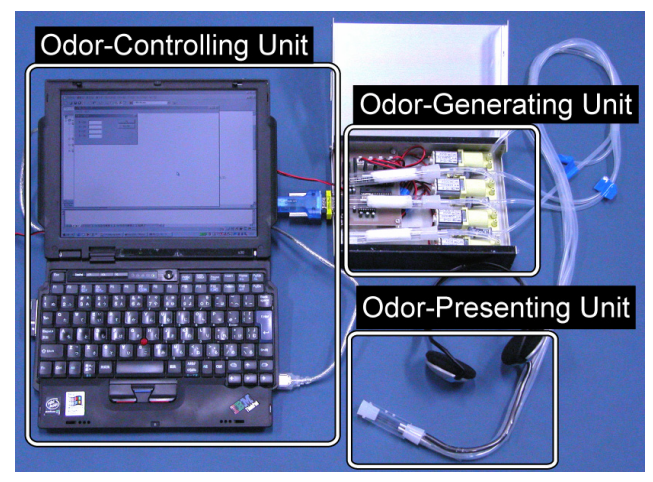

Fig.3 Photo of Wearable Olfactory Display

odor-controlling unit and odor-presenting unit. The configuration of this wearable olfactory display is shown in Fig. 2, and a photo of this wearable display is shown in Fig. 3. We constructed the odor-presenting system by combining the positioning system with this wearable olfactory display.

\subsection{Odor-Generating Unit}

The odor-generating unit is composed of air pumps and odor filters which are used for containing the perfume materials. Under resting conditions, the human respiratory volume is said to be about $6[1 / \mathrm{min}]$. Much of the breathing is done through the nose, except when the nose is blocked by, for example, nasal inflammation. This pump must generate an airflow nearly equal to the human respiratory volume, and be small and light enough to make it wearable. Therefore, we used a DC motor air pump (Enomoto Micro Pump Mfg.Co., CM-15) that meets the above requirements. The strength of the presented odor is controlled by changing the proportion of nonodor airflow to odor airflow. To generate and present three types of odor, we installed four air pumps, one for nonodor airflow and three for odor airflow. Pumps for odor airflow are connected to the odor filters with different perfume materials, and the pump for nonodor airflow is connected directly to the odor-presenting unit. We used silicon tubes for the connections, because silicon has only a faint smell and it is nontoxic to humans.

The odor filter is made of a test tube stuffed with cotton which is sufficiently infiltrated with perfume material. This increases the evaporation surface of the perfume material, therefore, nonodor 


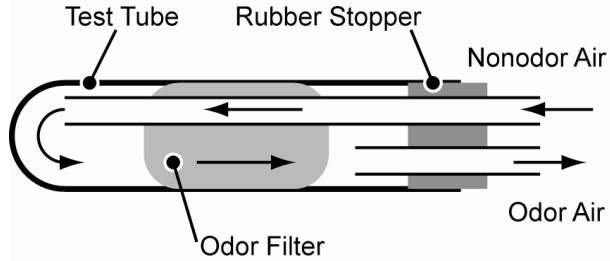

Fig.4 Structure of Odor Filter

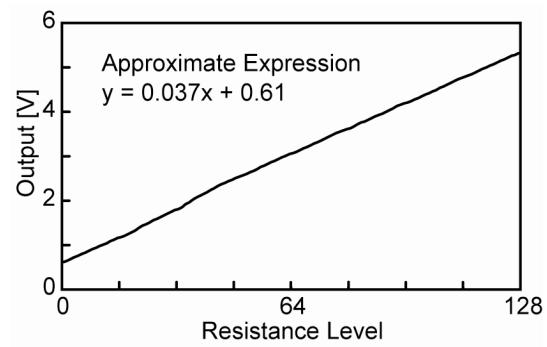

Fig.5 Relationship between Resistance Level of Potentiometer and Output Voltage to Air Pump

air easily changes into approximately saturated odor air. Fig. 4 shows the structure of the odor filter. In addition, we used perfume materials such as rosemary or lavender, which are available commercially for aroma therapy, and perfumed water for the odor source. These perfumed water and perfume materials are easy to evaporate, therefore nonodor air ventilating through the odor filter is rapidly changed into saturated odor air.

\subsection{Odor-Controlling Unit}

The odor-controlling unit is constructed of a notebook PC and a device controller. The device controller is composed of a microcomputer (Microchip Technology Co., PIC16F876) activated by $20[\mathrm{MHz}]$ clock frequency, a digital potentiometer, and a DC motor driver. This controller controls the air pump through voltage regulation using the digital potentiometer and the motor driver. This digital potentiometer is a variable-resistance element in which the resistance can be changed in 128 levels. When the input data to this element is 0 , resistance is set to level 1 on the 128-level scale and the volume of resistance becomes minimum. When the input data is 127 , resistance is set to level 128 on the 128 -level scale and the volume of resistance becomes maximum. We used high-speed serial communication to increase the time resolution, and we did not use PIC pulse-width modulation control in consideration of noise countermeasure and accuracy of control.

The notebook PC is used to calculate the strength of the presented odor using the data input from the positioning system, which will be described later in detail, and to output data for the odor-generating unit. The calculated and set output data are transferred to the device controller through a USB cable. The input data is the user's position and the output data includes the selected air pump and the airflow volume. The device controller controls the output voltage to the air pump using the data for the potentiometer sent from the PIC, which is based on the output data from the notebook $\mathrm{PC}$, to set the potentiometer resistance level in the range from 1 to 128. The relationship between the resistance level of the potentiometer and the output voltage to the air pump is shown in Fig. 5. From the measured result, the relationship can be approximated by a linear expression, as we

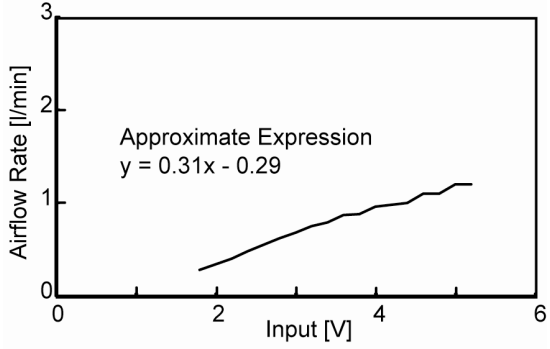

(a) Air Pump with Odor Filter

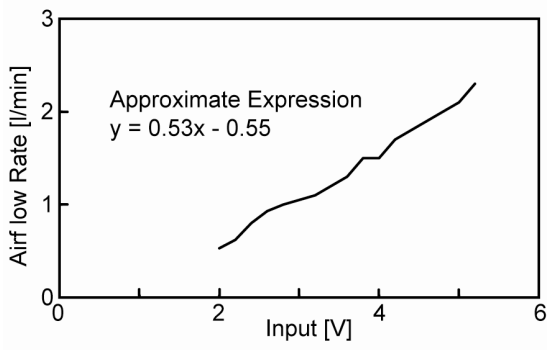

(b) Air Pump Only

Fig.6 Relationship between Airflow and Input Voltage of Air Pump

expected, and the functional output voltage was 0.62 [V] to 5.32 [V].

We also measured the relationship between the voltage applied to the air pump by the device controller and the airflow generated by the air pump. We took measurements of two settings. One was using the air pump with an odor filter; this setting is used for generating odor air. The other was using only the air pump; this setting is used for generating nonodor air. The measurement results are shown in Fig. 6. With the former setting, the air pump was started at the applied voltage of $1.8[\mathrm{~V}]$ and at that time, the airflow was $0.28[1 / \mathrm{min}]$. When $5.3[\mathrm{~V}]$, the maximum voltage of this system, was applied, the airflow was $1.3[1 / \mathrm{min}]$. With the latter setting, the air pump started at the applied voltage of 1.8 [V] and at that time, the airflow was $0.47[1 / \mathrm{min}]$. When $5.3[\mathrm{~V}]$, the maximum voltage of this system, was applied, the airflow was 2.4 $[1 / \mathrm{min}]$. On the basis of these results, the relationship between applied voltage and airflow was approximated by a linear expression. Because the range of the digital potentiometer level was 29 to 128 in the operation range of the applied voltage to the air pump, the airflow can be controlled in 100 steps.

Additionally, we measured the response time by measuring the time from the point of sending the output signal from the notebook PC to when the air is emitted from the odor-presenting unit. In this measurement, we placed a small piece of paper on the air emission hole and we defined the response time as the time between entering the data and when the piece of paper moved. We measured the response time by analyzing a movie taken with a camera at 30 [fps]. The result was a response time less than 50 [ms].

\subsection{Odor-Presenting Unit}

The mixing of odor air is accomplished in the odor-presenting unit. By mixing the odor air close to the user's nose, it is possible to reflect quickly the change of the air contents by airflow control and to quickly switch the odor. The structure of the odorpresenting unit is shown in Fig. 7. To reduce susceptibility to disturbances such as wind, we positioned the odor-presenting unit as close as possible to the user's nose. Also, to reduce any 


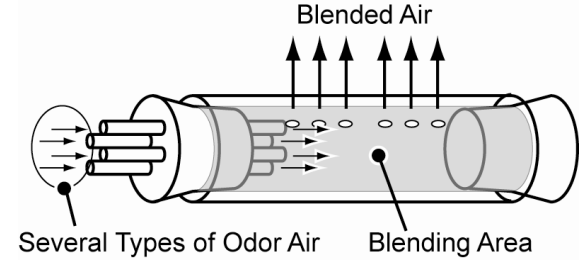

Fig.7 Odor-Presenting Unit

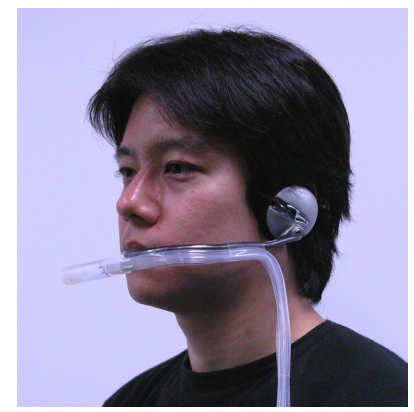

Fig.8 Wearing Odor-Presenting Unit

uncomfortable feeling of wearing the unit and to minimize the interruption of other information presentation or work operation, we selected neckband-type equipment for the odor-presenting unit. The appearance of wearing the odor-presenting unit is shown in Fig. 8 .

\subsection{Positioning System}

We constructed an odor field presentation system, which makes it possible to experience an odor field in a wide space, by combining the wearable olfactory display and the wide-space positioning system. We used the RFID tag space [16] for the wide-space positioning system. This system can detect the user's position and gain the position data through the RFID tag reader (RF Code CO., Spider) which reads the ID of the RFID buried in a reticular pattern at intervals of $1.2[\mathrm{~m}]$. The accuracy of the position information provided by this system is $600[\mathrm{~mm}]$. By changing the strength of odor presented from the wearable olfactory display according to the position data read by the tag reader, we can present an odor field in a wide space. The configuration of the odor field presentation system is shown in Fig. 9.

\section{Evaluation of Prototype 1}

We evaluated the prototype wearable olfactory display. The function needed to present the odor field, which is the aim of this work, is to grade the odor strength. To measure the odor strength, we used a portable odor meter (Futaba Electronics Co., FPO-II). This odor meter has two different gas sensors and outputs two patterns of data: raw output data from the two sensors and data of the strength and type of odor calculated from the output data of the two sensors. In the latter pattern of data, the strength of the odor is proportional to the vector length determined by mapping the output data of the two sensors on a plane, and the type of odor corresponds to the oblique angle [deg] of the vector.

\subsection{Evaluation of Odor Strength Control}

We evaluated the possibility of presenting graded odor strength, by using the odor meter. The result of the measurement is shown in Fig. 10(a). We used rosemary aroma oil as the perfume material. The top graph shows the airflow generated by the air pump with

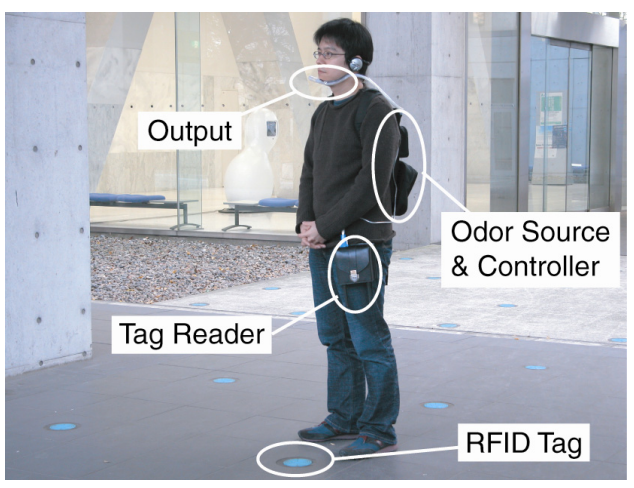

Fig.9 Configuration of Odor Field Presentation System
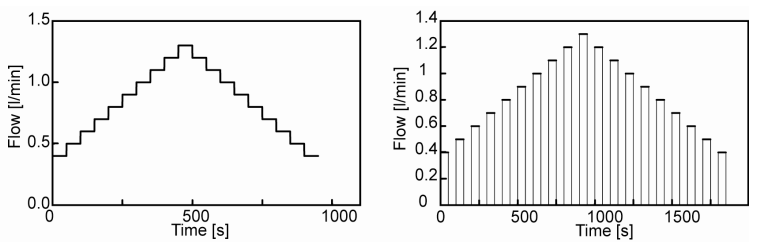

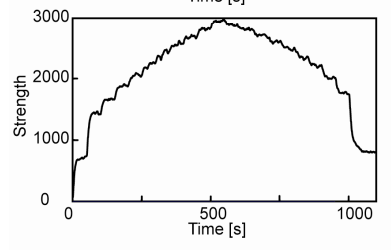

(a) Presentation Pattern 1

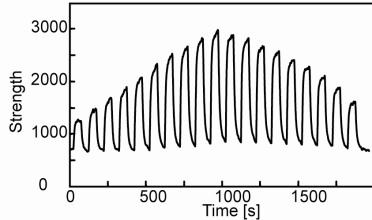

(b) Presentation Pattern 2
Fig.10 Results of Odor Strength

the odor filter used for generating the odor air. The bottom graph shows the odor strength measured with the odor meter. To avoid the odor meter becoming insensitive to the odor, we changed the airflow of the pump every 50 [s]. It is seen in the graph that the change of the airflow and the odor strength were related. However, because the odor meter became insensitive to the odor at a later time (after $500[\mathrm{~s}]$ ), at the same airflow, the odor meter detected a much stronger odor strength compared with that at an earlier time (before 500 [s]).

To confirm the reproducibility, taking into account that the odor meter became insensitive to the odor, we performed another experiment in which a nonodor state was held for 50 [s] before changing the odor strength. The result is shown in Fig. 10(b). It is seen from the graph that the odor strength during the nonodor term increased by small degrees. On the basis of this result, the difference between the odor strengths in the odor-presenting period and in the nonodor period is judged to be the actual strength of the presented odor. The above-described results prove that the prototype wearable olfactory display has controllability of odor strength.

\subsection{Evaluation of Odor Type Control}

We evaluated the odor type switching performance of the system. In this measurement, we used rosemary, lavender and orange aroma oils as the perfume material for the odor filter. We presented the odor in the rotation of nonodor, rosemary, lavender, and orange for $50[\mathrm{~s}]$ each. The results of the measurement using the odor meter with respect to the time variation of the strength and type of odor, and the raw output data from the two built-in sensors, are shown in Fig. 11.

Fig. 11(a) shows the time variation of the odor strength and the odor type measured with the odor meter. The output data was 


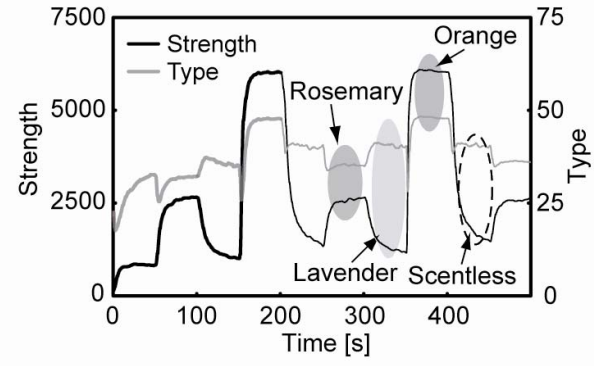

(a) Time Variation of Odor Strength and Type

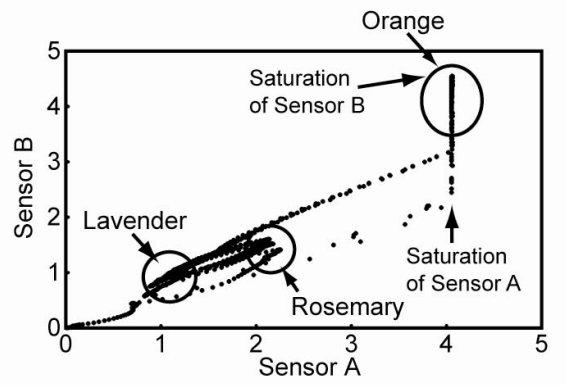

(b) Raw Output Data from Two Sensors

Fig.11 Results of Odor Switching

unstable for 250 [s] from the start of measurement, but after 250 [s], the output data changed periodically every 50 [s] according to the type of presented odor. From 250 [s] to 300 [s], the odor air of rosemary was presented, the odor air of lavender was presented from $300[\mathrm{~s}$ ] to $350[\mathrm{~s}]$, the odor air of orange was presented from 350 [s] to 400 [s], and nonodor air was presented from 400 [s] to 450 [s]. Shortly after each switch of odor air, a delay of the output data arose because the odor meter had become accustomed to the previous odor, as discussed in 4.2. However, the characteristic output data was shown for each odor air during its presentation period.

The graph in Fig. 11(b) shows the raw output data of the two built-in sensors, which were mapped on a plane. The data congregated at three points, and these points were in accord with the data for the static state of rosemary, lavender and orange. Incidentally, the dots between those three points were the knickpoints of switching the odor air. The above-described results prove the odor type switching capability of the prototype wearable olfactory display.

\section{FORMING AN OdOR FIELd}

An odor field is the distribution of odor strength in space, which varies with time. The time variation of the odor field is due to the diffusion of odor molecules vaporized from the odor source. In this research, we used an odor field that was defined by a simulation based on a diffusion equation for odor presentation.

\subsection{Odor Field in Static State}

First, we hypothesized that in a sufficiently wide space, there are no disturbances, such as airstream. Under this assumption, it is obvious that there is no angle dependence of odor strength. Therefore, we discuss the odor field in a system of spherical coordinates with the point of origin at the odor source, where we set the distance from the point of origin as " $r$ " and the density of the odor molecules in time " $t$ " as " $u(r, t)$ ". Then, because there is no angle dependence, the form of the diffusion equation becomes formula (1). Solving this formula as the static state and adding the

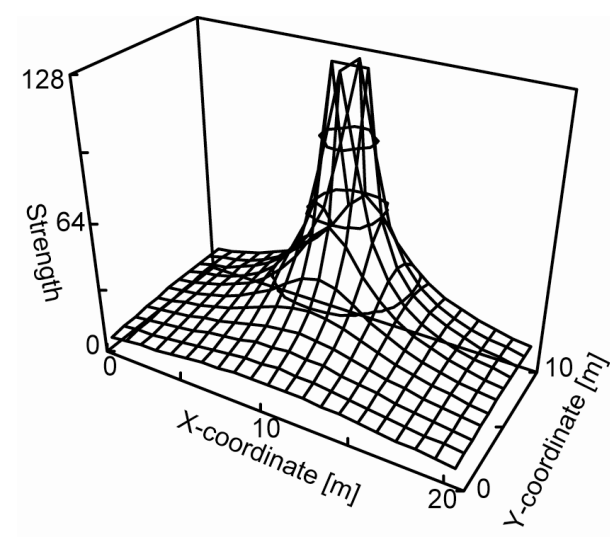

Fig. 12 3D Graph of Odor Field

condition of $\delta \mathrm{u} / \delta \mathrm{t}$, formula (1) becomes formula (2). Furthermore, from the condition that when $r$ goes to infinity $(r \rightarrow \infty) \mathrm{u}$ becomes zero $(\mathrm{u} \rightarrow 0$ ), formula (2) becomes formula (3). In this study, we use formula (3) to set up the odor field.

$$
\begin{gathered}
\frac{\partial u}{\partial t}=\alpha \frac{1}{r^{2}} \frac{\partial}{\partial t}\left(r^{2} \frac{\partial u}{\partial t}\right) \\
u=\frac{C_{1}}{r}+C_{2} \\
u=\frac{C}{r}
\end{gathered}
$$

Here, the constant "C" is the coefficient of the density of odor molecules which governs the strength of odor.

\section{Experiment of Odor Presentation}

We evaluated the odor field presentation performance of the constructed odor field presentation system in a wide space with an ambulant user. The evaluation was carried out by presenting the odor field described in section 5 to the subjects.

\subsection{Search for Odor Source}

The odor field was formed using formula (3). For the accuracy of $600[\mathrm{~mm}]$ of the positioning system, we sectioned the experimental space into $1[\mathrm{~m}]$ square grids and presented odor of various strengths depending on the grid position. In the experiment, we hypothesized that the odor source was located at the coordinates $(10,8)$. Fig. 12 shows the conceptual diagram of the presented odor field. The vertical axis (Strength) in Fig. 12 gives the resistance level of the digital potentiometer in the odorcontrolling unit. As described in 3.2, the relationship between the resistance level of this digital potentiometer and the airflow of odor air was approximately linear. Also, as described in 4.2, the strength of odor is related to the airflow volume of odor air. Therefore the resistance level of the digital potentiometer was used as the criterion for the odor strength.

In the experiment, we placed the odor source within the space of the outdoor positioning system $(18[\mathrm{~m}] \times 9[\mathrm{~m}])$ described in 3.4 and generated the odor field. The subject wearing the wearable olfactory display walked around searching for the odor source. This experiment of searching for the odor source was carried out only once for each subject with no time limit and each search ended when the subject claimed to have found the odor source.

The experiment was performed with four subjects (male and female, age late $10 \mathrm{~s} \sim$ early $20 \mathrm{~s}$ ) on the same day. To prevent the influence of seeing another subject's search, subjects were not 

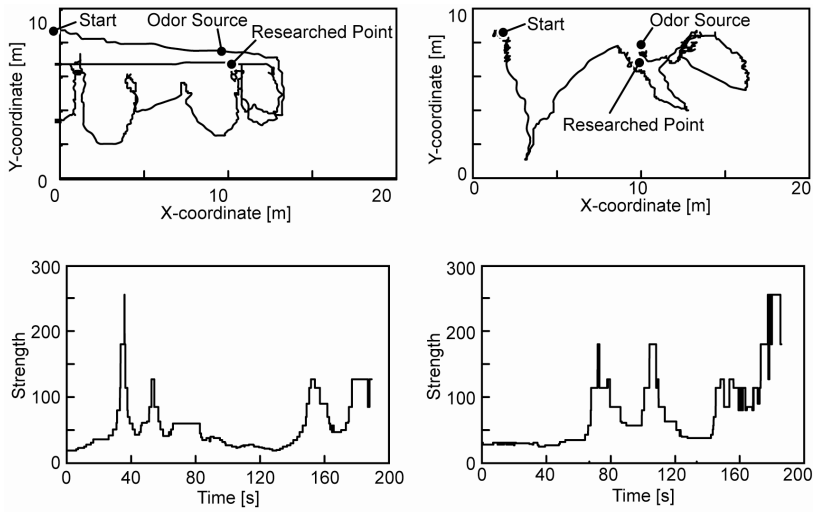

(a) Experimental Result of Subject C

Fig.13 Search Path and Presented Strength of Odor in Experimental Trial

permitted to watch the scene of another subject's search. Because the air pump for nonodor air was always running, the influence of the noise and vibration of the odor-generating unit was little. The weather was slightly windy and temperature was approximately $10\left[{ }^{\circ} \mathrm{C}\right]$.

Fig. 13 shows the typical results of the search for the odor source for two of the four subjects. The top graph shows the search route of the subject and the bottom graph shows the change of the odor strength presented to the subject during the search.

As seen from the graphs, both subjects reached the vicinity of the odor source by trial and error, changing their direction on the basis of the sensation of the changing odor. The result confirmed that the subject could perceive spatial information of odor presented by the prototype wearable olfactory display.

The method of search fell into two types: the scan type and the hill-climbing type. Subject $\mathrm{C}$ chose the strongest point of odor after searching through the entire experimental space. This method of search is the scan type. Subject D walked randomly and changed direction to that in which the perception of odor was stronger. This method of search is the hill-climbing type.

Additionally, many subjects had the impression that because the wind was strong, it was difficult to sense the odor. Consequently, it is necessary to discuss ways of avoiding the influence of wind and the uncomfortable feeling when wearing the display.

\section{Direct-INJECTION Wearable OlFactory Display (Prototype 2)}

The odor-generating unit of prototype 1 is constructed of pumps and odor filters. Using this equipment, pump and odor filters are needed to present every odor type. Therefore the more odor types we want to present, the larger the odor-generating unit becomes. Therefore, we constructed another type of wearable olfactory display (prototype 2) which can remain small even if the number of odor types is increased.

Prototype 2 is constructed of three units: the odor-presenting unit using an inkjet head device, the breath-detecting unit which is used to detect the user's breathing pattern, and the control unit. We call this olfactory display the "direct-injection wearable olfactory display".

The method of odor presentation is to inject odor droplets directly to or near the user's nose. In the odor-presenting unit with the inkjet head device, the perfume material is stocked in the device in liquid form and a pump becomes unnecessary. Therefore, the olfactory display can be made small. Additionally, by injecting

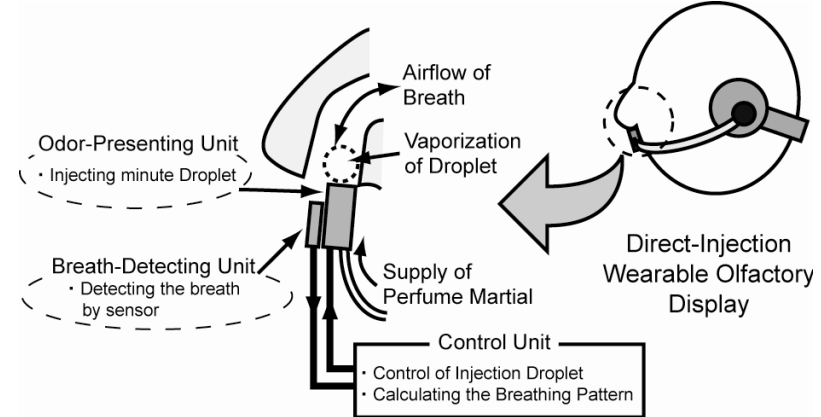

Fig.14 Schematic Diagram of Direct-Injection Wearable Olfactory Display

the minute odor droplet directly to the user's nose, subtle control of the odor strength and a high response of odor presentation become possible.

We suggested the idea of including the breath-detecting unit in the olfactory display. Many of the predecessor olfactory displays generated odor air full-time, so the odor air spread widely into the environment unnecessarily and influenced not only the olfactory display user but also other people nearby. We therefore proposed to present the odor air only when the user inhaled, by detecting the user's breathing pattern. This can reduce the amount of odor air used in vain when the user exhales and the spread of odor air into the environment. This will conserve the perfume material used for odor presentation. The perfume material used for odor presentation is a consumable material just the same as ink for a printer. Therefore the perfume material must be replaced or refilled. From this viewpoint, saving on the consumption of perfume material so that the wearable olfactory display can be used for a longer period is one of the important issues concerning the wearable olfactory display. The schematic diagram of the direct-injection wearable olfactory display is shown in Fig. 14

\subsection{Odor-Presenting Unit}

In the odor-presenting unit, we used MicroDrop (Microdrop Co., MD-K-130) as the inkjet head device to actualize the direct injection. MicroDrop is a piezotype inkjet head device which injects the droplet by applying a pressure pulse of the piezoelement to the tubule filled with the liquid. With this inkjet head device, we inject a droplet of perfume material directly to the user to present the odor. Additionally, this inkjet head device has a fast response time of $300[\mu \mathrm{s}]$.

\subsection{Breath-Detecting Unit}

In the breath-detecting unit, we used a temperature sensor (National Semiconductor Co., LM35) to detecting the user's breath. The method of perceiving the user's breathing pattern is to detect the change of temperature around the sensor caused by breathing through the nose. The temperature sensor in this unit is engulfed by resistors, therefore, in the steady state, the output from the sensor is stabilized at a certain value by the heat of the resistor's power consumption. Upon exhaling through the nose, the temperature around the sensor goes down and the sensor output changes. By measuring this change, the olfactory display detects the user's breathing pattern and presents the odor during the inhalation period.

\subsection{Control Unit}

In the control unit, the user's breathing pattern is calculated using the data from the breath-detecting unit, and the result of the 
calculation is employed to control the odor presentation. The control unit is composed of a notebook PC and a device controller, as in prototype 1 .

\section{Evaluating the Prototype 2 System}

First, we evaluated the function of each component part of the direct-injection wearable olfactory display, that is, the odorpresenting unit and the breath-detecting unit.

\subsection{Odor-Presenting Unit}

First, we measured the strength of the presented odor. In doing so, we compared two methods of injection. One is to inject the perfume material directly to the user's nose ("direct method"). The other method is to inject the perfume material to a reflecting plate placed just below the user's nose, which evaporates the perfume material ("indirect method"). Schematics of the direct method and the indirect method are shown in Fig. 15(b) and Fig. 15(c). In this measurement, we used yuzu orange aroma oil as the perfume material.

To measure the odor strength, we used a fragrance sensor (Shibaura Systems Co., SF-105A). This fragrance sensor has a quartz crystal, the characteristic frequency of which changes according to the mass of odor molecules adsorbed on its surface. By measuring this change in the characteristic frequency, the odor strength is determined. The relationship between the variation of the characteristic frequency and the odor strength is linear. Therefore, in this section, we use the measured value of the sensor, that is, the variation of the characteristic frequency, for the comparison of the odor strength.

Additionally, for the measurement of the odor strength, we prepared a model of the nasal fossa. This nasal fossa model was based on the nasal fossa model of Zarniko [17] and on actual measured values of a nasal fossa [18]. The schematic diagram of our nasal fossa model is shown in Fig. 16. We constructed the odor-measuring device by incorporating the fragrance sensor into

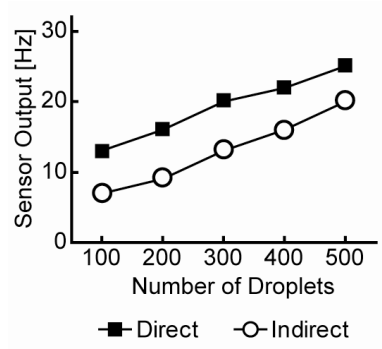

(a) Relationship between Number of Droplets and Smell strength

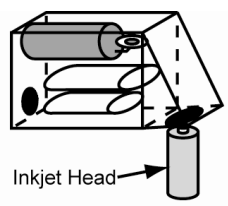

(a) Direct Injection

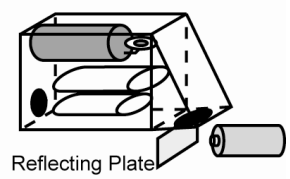

(b) Indirect Injection

Fig.15 Result of Measuring Odor Strength

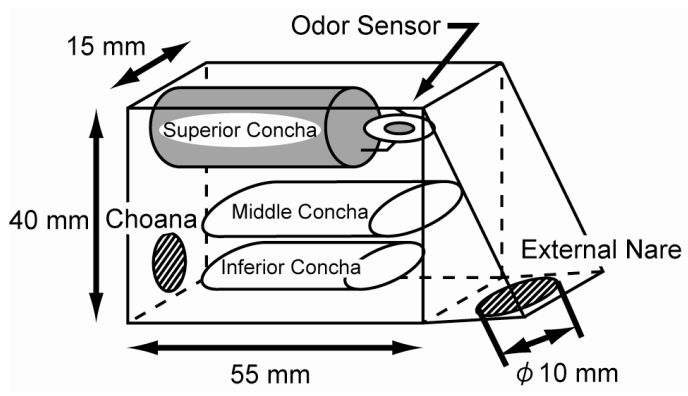

Fig.16 Model of Nasal Fossa

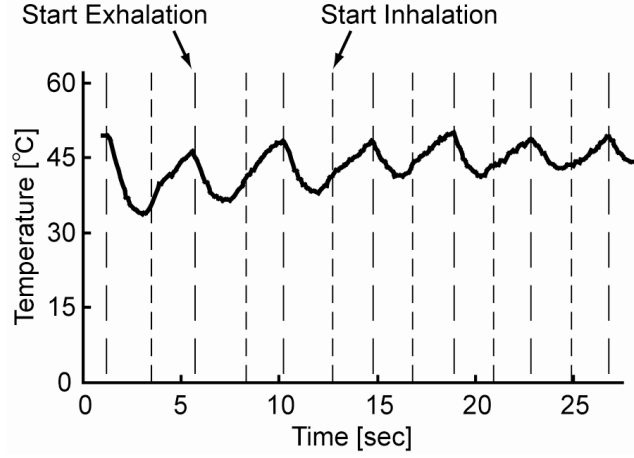

Fig.17 Result of Breath Detection

the nasal fossa model. Moreover, to re-create human inhalation, we generated $10[1 / \mathrm{min}]$ airflows in the model using a smallcapacity air pump (TECHNO TAKATSUKI CO., SPP-25GA). By assembling control circuits by which to switch the power source of the air pump on and off, we re-create inhalation in 3 [s] cycles.

The experimental results of odor strength presented directly and indirectly are shown in Fig. 15(a). It is seen that the odor strength can be controlled by controlling the number of droplets injected from the inkjet head device. The graph shows that direct injection presented a greater odor strength than did indirect injection. However, in several cases, droplets that were not evaporated were observed in both direct and indirect methods. It is conceivable that a greater odor strength will be possible upon evaporating all these droplets. Considering these results, we will investigate a way of completely evaporating the injected droplets of perfume material.

\subsection{Breath-Detecting Unit}

We experimented on detecting the human breath using the breathdetecting unit with the temperature sensor. The results of this experiment are shown in Fig. 17 as the change of the temperature around this sensor. In this experiment, the sensor was placed about $1[\mathrm{~cm}]$ below the subject's nose and the subject's breathing was that of the resting state. The start time of the exhalation period was detected with a sufficiently short response time of less than 50 [ms]. The start time of the inhalation period was found to be detectable by focusing on the point at which the variation of the output data from the sensor changes to a positive value from a negative value. We expected the variation of the output data of the sensor to be a negative value during the exhalation period because by exhaling, hot air around the sensor will be blown away, causing the temperature to drop. We also expected the changing point to be detected at the start time of the inhalation period. However, the results show that the changing point was about 500 [ms] earlier than the start of the inhalation period. We considered that this time lag corresponds to the nonbreathing period which is the relaxation period of the muscles used for breathing. Therefore, we considered that it is possible to calculate the start time of the inhalation period by focusing on the changing point and setting the start time to be $500[\mathrm{~ms}]$ after the changing point.

\section{Comparison of Prototype 1 and Prototype 2}

We compared the basic function of prototype 1 and prototype 2: the operation duration of the wearable olfactory display.

\subsection{Operation Duration of Wearable Olfactory Display}

First, we pooled $0.05[\mathrm{ml}]$ of perfume material into the odor filter for prototype 1 and ran prototype 1 continuously to measure the alteration of the presented odor strength. The results are shown in 


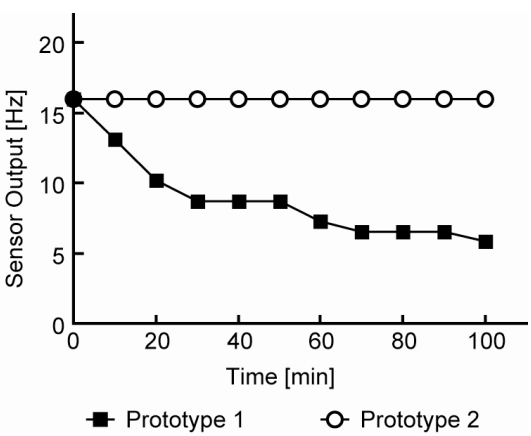

Fig.18 Alteration of Odor Strength

Fig. 18. The odor strength was measured at ten-minute intervals with the odor-measuring device using the model of the nasal fossa. It was found that with 0.05 [ml] of perfume material, prototype 1 could present an odor strength of $16[\mathrm{~Hz}]$ at maximum. It was also revealed that the duration of presenting an odor strength of 16 $[\mathrm{Hz}]$ was less than 10 minutes.

Second, using prototype 2, we examine presenting an odor strength of $16[\mathrm{~Hz}]$, which was the maximum strength for prototype 1 using 0.05 [ml] of perfume material. Referring to the experiment results described in 8.1, the direct-injection olfactory display prototype 2 could realize the presentation of an odor strength of 16 [Hz] with 200 droplets by the direct method and 400 droplets by the indirect method. Because the volume of a droplet from the inkjet head device is approximating 200 [pl], odor presentation was possible more than 600 times using 0.05 [ml] of perfume material. Estimating that a person breathes 10 times a minute, continuous odor presentation for more than 60 minutes is possible. Therefore, compared with prototype 1, prototype 2 could present a greater odor strength with the same volume of perfume material.

On the basis of the above results, with the same volume of perfume material, we found that it is possible to use prototype 2 for a longer duration than prototype 1 . That is, it is considered that prototype 2 has a more efficient consumption of perfume material and can also present a stable odor strength.

\section{Conclusion ANd Future Work}

In this paper, we discussed an olfactory display which can be used in various environments such as outdoors or in a wide space. First, we constructed the prototype 1 wearable olfactory display which was portable and robust against environmental factors. We evaluated the performance of prototype 1 for controllability of the strength and type of odor to be presented. We also devised an odor-field-presenting system which presents spatial information of odor outdoors, using an RFID tag space as the positioning system, and evaluated it by presenting the odor field in a real space. The results of the evaluation revealed that it is possible to identify the odor source using the odor field, and it is also possible to present the spatial spread of odor in real space.

Second, we constructed prototype 2 as an improvement of prototype 1 . We focused particularly on the reduction in size and weight and long operation life. We proposed the use of an inkjet head device for presenting the odor, as well as the detection of the user's breathing using a temperature sensor. We constructed a wearable olfactory display with the inkjet head device in the odorpresenting unit and a breath-detecting unit with a temperature sensor and evaluated the function of each unit. The results of the evaluation proved the feasibility of the direct-injection wearable olfactory display and its superior operation life and stability in presenting odor strength over prototype 1 .
In future, we will incorporate a positioning system into prototype 2 and construct an odor-field-presenting system. Additionally, by applying the function of detecting the user's breathing, we propose to endow interactivity to the olfactory display, which most existing olfactory displays do not have. Basically, in the act of smelling, humans perform the action called "sniffing" to inhale more air. By detecting this "sniffing" action, we hope to realize an interactive olfactory display.

\section{REFERENCE}

[1] T. Nakamoto, S. Utsumi, N. Yamashita, T. Moriizumi and Y. Sonoda, "Active Gas Sensing System Using Automatically Controlled Gas Blender and Numerical Optimization Technique," Sensors and Actuators B, 20 (1994) 131.

[2] H. Troy Nagle, Susan S. Schiffman and Ricardo Gutierrez-Osuna1, "The How and Why of Electronic Noses," IEEE Spectrum, Vol.35, No.9, pp.22-31, 1998.

[3] N.A.Rakow and K.S.Suslick, "A colorimetric sensor array for odour visualization," Nature, v406, p.710-713, 2000.

[4] Joseph Nathaniel Kaye, "Symbolic Olfactory Display," Master Thesis for Media Arts and Sciences School of Architecture and Planning, MIT, May 2001.

[5] M.L.Heilig, "El cine del futuro: The cinema of the future," Presence, Vol.1, No.3, pp.193-219, 1992.

[6] M.Zybura and G.A.Eskeland, "Olfaction for Virtual Reality," Quarter Project, Industrial Engineering 543, University of Washington, 1999.

[7] france telecom, http://www.rd.francetelecom.com/en/galerie/senteurs _multimedia/doc_avance.php

[8] Digiscents, "iSmell", http://www.contourinc.com/p/prod15.html

[9] NCR, Web KIOSK, http://www.ncr.co.jp/products/hardware/webkio sk/aroma.html

[10] AromaJet, E-Commerce Kiosk, http:// www.aromajet.com

[11] T. Nakamoto, Y. Nakahira, H. Hiramatsu and T. Moriizumi, "Odor recorder using active odor sensing system," Sensors and Actuators B, 76(2001) 465.

[12] T. Yamanaka, T. Nakamoto, "Improvement of Odor-Recorder Robustness against Environmental Change using Real-time Reference Method," The transactions of the Institute of Electrical Engineers of Japan E, Jun. 2002, pp.312-317. 2002. (in Japanese)

[13] S. Sakikawa, T. Tanikawa, K. Hirota, M. Hirose, "A Study of Transmission of Olfactory Reality," Transactions of the Virtual Reality Society of Japan, Vol.7, 2002. (in Japanese)

[14] M. Hirose, T. Tanikawa, S. Tanaka, S. Sakikawa, "A Study of Olfactory Display," Transactions of the Virtual Reality Society of Japan, Vol.5, pp.193-196, 2000. (in Japanese)

[15] Y. Yanagida, S. Kawato, H. Noma, A. Tomono, and N. Tetsutani: "Projection-Based Olfactory Display with Nose Tracking," Proc. of IEEE Virtual Reality 2004, pp.43-50, 2004.

[16] T. Amemiya, J. Yamashita, K. Hirota, M. Hirose: "Virtual Leading Blocks for the Deaf-Blind : A Real-Time Way-Finder by VerbalNonverbal Hybrid Interface and High-Density RFID Tag Space" Proc. of IEEE Virtual Reality 2004, pp.165-172, 2004.

[17] Zarniko.C: "Die Krankheiten der Nase des Nasenrachens," Karger, Berlin,

[18] H. Sakakibara, A. Ishida, T. Nakai, H. Suzuki, "3-D FEM Analysis of Realistic Model of Nasal Cavity Observed by MRI," Technical report of IEICE. EA, Vol.95, No.14, pp.21-28, 1995. (in Japanese) 